\title{
Laparoscopic gastrectomy after incomplete endoscopic resection for early gastric cancer
}

\author{
HIRONORI TSUJIMOTO, YOSHIHISA YAGUCHI, ISAO KUMANO, RISA TAKAHATA, \\ YUSUKE MATSUMOTO, KAZUMICHI YOSHIDA, HIROYUKI HORIGUCHI, SUEFUMI AOSASA, \\ SATOSHI ONO, JUNJI YAMAMOTO and KAZUO HASE
}

Department of Surgery, National Defense Medical College, 3-2 Namiki, Tokorozawa 359-8513, Japan

Received December 2, 2011; Accepted January 23, 2012

DOI: $10.3892 / o r .2012 .2046$

\begin{abstract}
Endoscopic submucosal dissection (ESD) utilizes electrical coagulation, which can cause burns, fibrosis and adhesion of the stomach and surrounding tissue; these complications might increase the surgical difficulties for subsequent laparoscopy-assisted gastrectomy (LAG) and the risk of complications. However, scarce data are available on the influence of previous ESD on LAG. The purpose of this study was to evaluate the feasibility and safety of LAG following incomplete ESD in patients with early gastric cancer. Ninety-seven patients who underwent LAG were analyzed retrospectively; 17 patients had undergone ESD previously and the remaining 80 patients had no history of ESD. Clinicopathological data and surgical outcomes were compared between the two groups. No differences were observed in surgical outcomes of LAG after ESD in terms of operation time, intraoperative blood loss, total number of harvested lymph nodes, time until start of flatus, and postoperative hospital stay. These results were not influenced by tumor location and operative procedures. In conclusion, in terms of surgical outcomes, LAG is a safe and feasible procedure for the treatment of early gastric cancer regardless of previous endoscopic treatment. LAG may be the first-choice radical treatment after incomplete ESD for early gastric cancer.
\end{abstract}

\section{Introduction}

Advances in diagnostic techniques have increased the detection rate of small and early-stage gastric cancers $(1,2)$. The incidence of early gastric cancer is more than $40 \%(3,4)$, and patients with early gastric cancer have an extremely favorable prognosis after curative treatment, with 5-year survival rates

Correspondence to: Dr Hironori Tsujimoto, Department of Surgery, National Defense Medical College, 3-2 Namiki, Tokorozawa 359-8513, Japan

E-mail: tsujihi@ndmc.ac.jp

Key words: early gastric cancer, incomplete endoscopic resection, laparoscopic gastrectomy exceeding 90\% (4-6). Endoscopic resection (ER), including endoscopic mucosal resection (EMR) and endoscopic submucosal dissection (ESD), may be optimal for early gastric cancer in terms of improving the quality of life. However, ER sometimes fails to completely remove the cancerous lesion, and pathological examination of the resected specimen occasionally reveals a potentially high risk of lymph node metastases that does not meet the criteria for curative ER (7). These are considered cases of incomplete ER and additional ER and/or radical gastrectomy should be performed for such cases.

Since Kitano et al (8) reported the first laparoscopyassisted gastrectomy (LAG) for gastric cancer in 1991, this technique has become increasingly popular. The advantages of LAG include reduced blood loss and pain, early recovery of digestive tract activity, and short hospital stay $(9,10)$. Therefore, LAG has been regarded as a standard procedure for treatment of early gastric cancer.

ER-induced inflammation causes edema, fibrosis, and adhesion of the stomach and surrounding tissue, which might increase the surgical difficulties for subsequent LAG and the risk of complications; however, few data are available on the influence of previous ER on LAG (11).

In this study, to assess the feasibility and safety of LAG after ER, we reviewed surgical outcomes for patients who underwent ESD and subsequently underwent LAG for early gastric cancer.

\section{Materials and methods}

Patients. One hundred-nine patients with early gastric cancer underwent LAG at the Department of Surgery from 2008 to 2010. Twelve patients who underwent simultaneous resection of other organs, such as the gall bladder (gallstones) and sigmoid colon (cancer), were excluded from this study. Seventeen patients were primarily assigned to the ESD group according to the gastric cancer treatment guidelines in Japan (7); among these 17 patients, 4 experienced active bleeding during endoscopic resection and the procedure was stopped. These patients subsequently underwent LAG. The remaining 13 patients underwent ESD. Indications for further LAG in these 13 patients were positive ESD margin (9 patients) and positive lymphatic and/or venous involvement in the ESD specimen (4 patients). The average interval between LAG and 
Table I. Demographic data in patients with or without previous endoscopic treatment.

$\begin{array}{ccc}\text { Previous } & \text { No previous } & \\ \text { ESD } & \text { ESD } & \text { P-value } \\ \end{array}$

Number
Age (years)
Gender
Male
Female

$\operatorname{BMI}\left(\mathrm{kg} / \mathrm{m}^{2}\right)$

17

80

Tumor location

$$
\text { U }
$$

M

L

Tumor circumference

Ant
Post
Less
Gre

3 (17.6)

4 (23.5)

$10(58.8)$

$0(0.0)$

Macroscopic type

Elevated

Depressed

7 (41.2)

$8(10.0)$

3 (17.6)

Mixed

7 (41.2)

$65(81.3)$

7 (8.8)

Maximal tumor size (mm)

$21.5 \pm 9.0$

$37.6 \pm 23.8$

Histological classification

Intestinal
Diffuse

$17(100.0)$

49 (61.3)

$0(0.0)$

$31(38.8)$

Tumor depth

T1a

T1b1

T1b2

$\mathrm{T} 2$

T3

$\mathrm{T} 4 \mathrm{a}$

Lymph node metastasis

$\begin{array}{ccc}\text { N0 } & 17(100.0) & 70(87.5) \\ \text { N1 } & 0(0.0) & 8(10.0) \\ \text { N2 } & 0(0.0) & 2(2.5) \\ \text { Stage } & & \\ \text { IA } & 17(100.0) & 60(75.0) \\ \text { IB } & 0(0.0) & 10(12.5) \\ \text { IIA } & 0(0.0) & 5(6.3) \\ \text { IIB } & 0(0.0) & 3(3.8) \\ \text { IIIB } & 0(0.0) & 2(2.5)\end{array}$

Operative procedure

$\begin{array}{lcc}\text { LDG } & 7(41.2) & 42(52.5) \\ \text { LPPG } & 3(17.6) & 19(23.8) \\ \text { LPG } & 4(23.5) & 6(7.5) \\ \text { LTG } & 3(17.6) & 13(16.3)\end{array}$

0.40
Table I. Continued.

\begin{tabular}{lccc}
\hline & $\begin{array}{c}\text { Previous } \\
\text { ESD }\end{array}$ & $\begin{array}{c}\text { No previous } \\
\text { ESD }\end{array}$ & P-value \\
\hline $\begin{array}{l}\text { Lymphadenectomy } \\
\text { D1+ } \alpha\end{array}$ & $8(50.0)$ & $19(23.8)$ & 0.09 \\
D1+ $\beta$ & $8(50.0)$ & $43(53.8)$ & \\
D2 & $1(6.3)$ & $18(22.5)$ & \\
Total number of & $22.0 \pm 9.3$ & $27.0 \pm 14.2$ & 0.17 \\
harvested LNs & & & \\
\hline
\end{tabular}

BMI, body mass index; $\mathrm{U}$, upper third of the stomach; $\mathrm{M}$, middle third of the stomach; L, lower third of the stomach; Ant, anterior wall of the stomach; Post, posterior wall of the stomach; Less, lesser curvature; Gre, greater curvature; LDG, laparoscopic distal gastrectomy; LPPG, laparoscopic pylorus-preserving gastrectomy; LPG, laparoscopic proximal gastrectomy; LTG, laparoscopic total gastrectomy; LN, lymph node.

ESD was 44.8 \pm 19.0 days (range, 19-80 days) in patients with previous ESD. Eighty patients whose tumors did not meet the criteria for curative ER had no history of ESD and comprised the control group.

Clinicopathological findings of the patients were evaluated according to the Japanese Classification of Gastric Carcinoma (JCGC) (second English edition) published by the Japanese Gastric Cancer Association (12). Diagnosis of early gastric cancer was based on the preoperative assessment of depth of wall invasion by upper gastrointestinal tract endoscopy, barium radiology, and endoscopic ultrasonography, with nodal involvement determined by preoperative computed tomography (4).

Indication for and procedure of ESD. Since 2005, we have regarded the following features as indications for ESD according to the gastric cancer treatment guidelines in Japan (7): i) presence of differentiated-type carcinoma limited to the mucosal layer; and ii) absence of ulceration or ulcer scars irrespective of the macroscopic type. A single-channel endoscope (GIF-H260; Olympus, Tokyo, Japan) was inserted under conscious sedation. Lesions were marked beyond the margins using a conventional needle knife (Needle papillotome; MTW Endoscopy, Wesel, Germany). A solution of $0.25 \%$ sodium hyaluronate in normal saline solution containing $0.001 \%$ epinephrine and $0.002 \%$ indigo carmine was injected into the submucosal layer and a circumferential incision was made to include the markings. Lesions were dissected using an insulation-tipped electrosurgical knife (EMR Knife; MTW Endoscopy) to curatively exfoliate tumors through the submucosal layer.

Indication for and procedure of $L A G$. Indications for $\mathrm{LAG}$ were as per those of the gastric cancer treatment guidelines in Japan (7), i.e., clinically mucosal or submucosal carcinoma without lymph node metastasis (cT1, cN0) is an indication for LAG.

After induction of general anesthesia, each patient was placed in a supine position. The surgeon stood on the left side 
Table II. Surgical outcomes in patients with or without previous endoscopic treatment.

\begin{tabular}{|c|c|c|c|}
\hline & Previous ESD & No previous ESD & P-value \\
\hline Number & 17 & 80 & \\
\hline \multicolumn{4}{|l|}{ Co-morbidity } \\
\hline No & $5(29.4)$ & $48(60.0)$ & \multirow[t]{7}{*}{0.02} \\
\hline Yes $^{\mathrm{a}}$ & $12(70.6)$ & $32(40.0)$ & \\
\hline Hypertension & $8(47.1)$ & $14(17.5)$ & \\
\hline Diabetes & $2(11.8)$ & $8(10.0)$ & \\
\hline Respiratory dysfunction & $1(5.9)$ & $4(5.0)$ & \\
\hline Arrhythmia & $0(0.0)$ & $3(3.8)$ & \\
\hline Others & $2(11.8)$ & $6(7.5)$ & \\
\hline \multicolumn{4}{|l|}{ Previous laparotomy } \\
\hline No & $11(64.7)$ & $61(76.3)$ & \multirow[t]{2}{*}{0.32} \\
\hline Yes & $6(35.3)$ & $19(23.8)$ & \\
\hline \multicolumn{4}{|l|}{ Postoperative complications } \\
\hline No & $15(88.2)$ & $71(88.8)$ & \multirow[t]{8}{*}{0.95} \\
\hline Yes & $2(11.8)$ & $9(11.3)$ & \\
\hline Gastric fullness & $1(6.3)$ & $5(7.1)$ & \\
\hline Intestinal obstruction & $1(6.3)$ & $0(0.0)$ & \\
\hline Enteritis & $0(0.0)$ & $1(1.4)$ & \\
\hline Anastomotic leakage & $0(0.0)$ & $1(1.4)$ & \\
\hline Wound infection & $0(0.0)$ & $1(1.4)$ & \\
\hline Afferent loop syndrome & $0(0.0)$ & $1(1.4)$ & \\
\hline Operation time (min) & $216.8 \pm 33.5$ & $217.6 \pm 41.5$ & 0.94 \\
\hline Intraoperative bleeding (ml) & $54.8 \pm 34.7$ & $51.6 \pm 56.0$ & 0.82 \\
\hline Time until the first flatus (days) & $2.9 \pm 1.1$ & $2.7 \pm 0.8$ & 0.44 \\
\hline Time until start of oral intake (days) & $4.1 \pm 2.0$ & $4.0 \pm 2.7$ & 0.87 \\
\hline Hospital stay (day) & $13.2 \pm 10.2$ & $12.4 \pm 10.4$ & 0.78 \\
\hline Conversion to open surgery & $0(0)$ & $0(0)$ & $>0.99$ \\
\hline
\end{tabular}

${ }^{\mathrm{a} T h r e e ~ p a t i e n t s ~ h a d ~ a ~ h i s t o r y ~ o f ~ t w o ~ o r ~ m o r e ~ c o-m o r b i d i t i e s . ~}$

of the patient and the first assistant stood on the right side; the laparoscopist stood between the abducted legs of the patient. A camera port was inserted into an inferior umbilical incision. Next, a pneumoperitoneum of $10-12 \mathrm{mmHg}$ was created, and four additional ports (two ports with a diameter of $12 \mathrm{~mm}$ and two with a diameter of $5 \mathrm{~mm}$ ) were inserted into the left upper, right lower, left lower, and right upper quadrants under laparoscopic imaging. An ultrasonically activated sealing device (Harmonic Scalpel Ace; Ethicon, Tokyo, Japan) and/or vessel sealing device (LigaSure V, Tyco Healthcare, Tokyo, Japan) was used.

Lymph node dissection of D1+ $\alpha$ indicated dissection of the perigastric lymph nodes and nodes along the left gastric artery (station 7). D1+ $\beta$ lymph node dissection indicated dissection of the perigastric lymph nodes and stations 7, 8a (anterosuperior group of the common hepatic artery) and 9 (celiac axis). D2 lymph node dissection indicated dissection of the perigastric nodes and all second-tier nodes, depending on the tumor location. The lymph node station number was classified according to JCGC (12). Either laparoscopic distal gastrectomy (LDG) or laparoscopic pylorus-preserving gastrectomy (LPPG) was indicated for tumors located in the middle or lower part of the stomach. After LDG, either Billroth I anastomosis or Rouxen-Y reconstruction was performed with a 4-cm upper midline incision, depending on the size of the remnant stomach. LPPG was performed for tumors in the middle third of the stomach located at least $5 \mathrm{~cm}$ proximal to the pyloric ring, followed by two-layer gastro-gastro anastomosis. Laparoscopic proximal gastrectomy (LPG) was indicated for tumors in the upper part of the stomach and more than half could be preserved as remnant stomach. Laparoscopic total gastrectomy (LTG) was performed for tumors in the upper third of the stomach by adapting LPG. After LPG and LTG, esophagogastrostomy and esophagojejunostomy were performed, respectively, using a linear stapler (ETS45; Ethicon, blue cartridge) as previously described with some modifications $(13,14)$. LAG was performed by a surgeon who had previously performed more than 50 LAGs.

Statistical analysis. Statistical calculations were performed using StatView version 5.0 (SAS Institute, Inc., Cary, NC, USA). The data are expressed as mean \pm SD. Statistical analyses were performed using the Mann-Whitney U test or $\chi^{2}$ test with Fisher's exact test, as appropriate. Univariate and multi- 
Table III. Surgical outcomes according to the surgical procedures in patients with or without previous endoscopic treatment.

\begin{tabular}{|c|c|c|c|c|c|}
\hline \multirow[b]{2}{*}{ Surgical procedures } & \multicolumn{2}{|c|}{ Previous ESD } & \multicolumn{2}{|c|}{ No previous ESD } & \multirow[b]{2}{*}{ P-value } \\
\hline & $\mathrm{N}$ & Mean \pm SD & $\mathrm{N}$ & Mean \pm SD & \\
\hline \multicolumn{6}{|l|}{ Operation time (min) } \\
\hline LDG & 7 & $201.4 \pm 33.9$ & 42 & $213.7 \pm 42.0$ & 0.47 \\
\hline LPPG & 3 & $212.7 \pm 9.0$ & 19 & $212.4 \pm 33.6$ & 0.99 \\
\hline LPG & 4 & $221.0 \pm 31.5$ & 6 & $219.3 \pm 51.1$ & 0.96 \\
\hline LTG & 3 & $251.3 \pm 35.9$ & 13 & $235.8 \pm 45.2$ & 0.59 \\
\hline \multicolumn{6}{|c|}{ Intraoperative blood loss (ml) } \\
\hline LDG & 7 & $60.3 \pm 38.0$ & 42 & $47.3 \pm 52.7$ & 0.54 \\
\hline LPPG & 3 & $42.7 \pm 15.3$ & 19 & $67.6 \pm 67.1$ & 0.54 \\
\hline LPG & 4 & $52.0 \pm 47.0$ & 6 & $40.3 \pm 21.0$ & 0.60 \\
\hline LTG & 3 & $58.0 \pm 37.2$ & 13 & $47.9 \pm 61.0$ & 0.79 \\
\hline \multicolumn{6}{|c|}{ Total numbers of harvested LNs } \\
\hline LDG & 7 & $18.3 \pm 10.0$ & 42 & $26.2 \pm 13.1$ & 0.13 \\
\hline LPPG & 3 & $22.7 \pm 1.5$ & 19 & $23.7 \pm 11.6$ & 0.88 \\
\hline LPG & 4 & $19.8 \pm 7.9$ & 6 & $17.2 \pm 10.2$ & 0.68 \\
\hline LTG & 3 & $33.0 \pm 7.2$ & 13 & $39.0 \pm 16.5$ & 0.56 \\
\hline \multicolumn{6}{|c|}{ Time until start of flatus (days) } \\
\hline LDG & 7 & $2.3 \pm 0.5$ & 42 & $2.7 \pm 0.8$ & 0.11 \\
\hline LPPG & 3 & $3.0 \pm 0.0$ & 19 & $2.5 \pm 0.7$ & 0.25 \\
\hline LPG & 4 & $2.8 \pm 1.0$ & 6 & $2.8 \pm 0.8$ & 0.88 \\
\hline LTG & 3 & $4.3 \pm 1.5$ & 13 & $2.8 \pm 1.1$ & 0.06 \\
\hline \multicolumn{6}{|c|}{ Time until start of oral intake (days) } \\
\hline LDG & 7 & $3.3 \pm 0.5$ & 42 & $4.2 \pm 3.6$ & 0.49 \\
\hline LPPG & 3 & $4.7 \pm 2.9$ & 19 & $3.7 \pm 1.2$ & 0.31 \\
\hline LPG & 4 & $3.8 \pm 1.0$ & 6 & $4.0 \pm 0.6$ & 0.63 \\
\hline LTG & 3 & $6.0 \pm 3.5$ & 13 & $3.7 \pm 1.6$ & 0.09 \\
\hline \multicolumn{6}{|c|}{ Postoperative hospital stay (days) } \\
\hline LDG & 7 & $8.4 \pm 1.3$ & 42 & $11.9 \pm 7.7$ & 0.25 \\
\hline LPPG & 3 & $22.7 \pm 22.9$ & 19 & $14.1 \pm 16.6$ & 0.44 \\
\hline LPG & 4 & $10.3 \pm 2.1$ & 6 & $10.7 \pm 4.8$ & 0.88 \\
\hline LTG & 3 & $18.7 \pm 4.2$ & 13 & $12.4 \pm 9.3$ & 0.28 \\
\hline
\end{tabular}

LDG, laparoscopic distal gastrectomy; LPPG, laparoscopic pylorus-preserving gastrectomy; LPG, laparoscopic proximal gastrectomy; LTG, laparoscopic total gastrectomy; LN, lymph node.

variate analyses were performed using the Cox proportional hazards model. P-values of $<0.05$ were considered statistically significant.

\section{Results}

The demographic data of patients who underwent LAG for early gastric cancer with or without previous ESD are depicted in Table I. Although the patient group that previously underwent ESD consisted of significantly more males than the group without previous ESD, no differences were observed in patient age, body mass index (BMI), tumor location and tumor circumference between the two groups. The patient group without previous ESD had a significantly higher incidence of depressed-type lesions, larger tumor size and a more frequent incidence of diffuse-type lesions because of the indications for ESD. No differences were observed in pathological stage, operative procedure, extent of lymphadenectomy and total number of harvested lymph nodes between the two groups.

Patients with previous ESD had co-morbidities more frequently than patients without previous ESD (Table II). No differences were observed in surgical outcomes such as incidence of postoperative complications, operation time, intraoperative blood loss, time until the start of flatus, time until the start of oral intake, and postoperative hospital stay between the two groups. No patient in either group was converted to open surgery. Next, we examined surgical outcomes in both groups according to the operative procedures (Table III). No differences were observed in operation time, intraoperative 


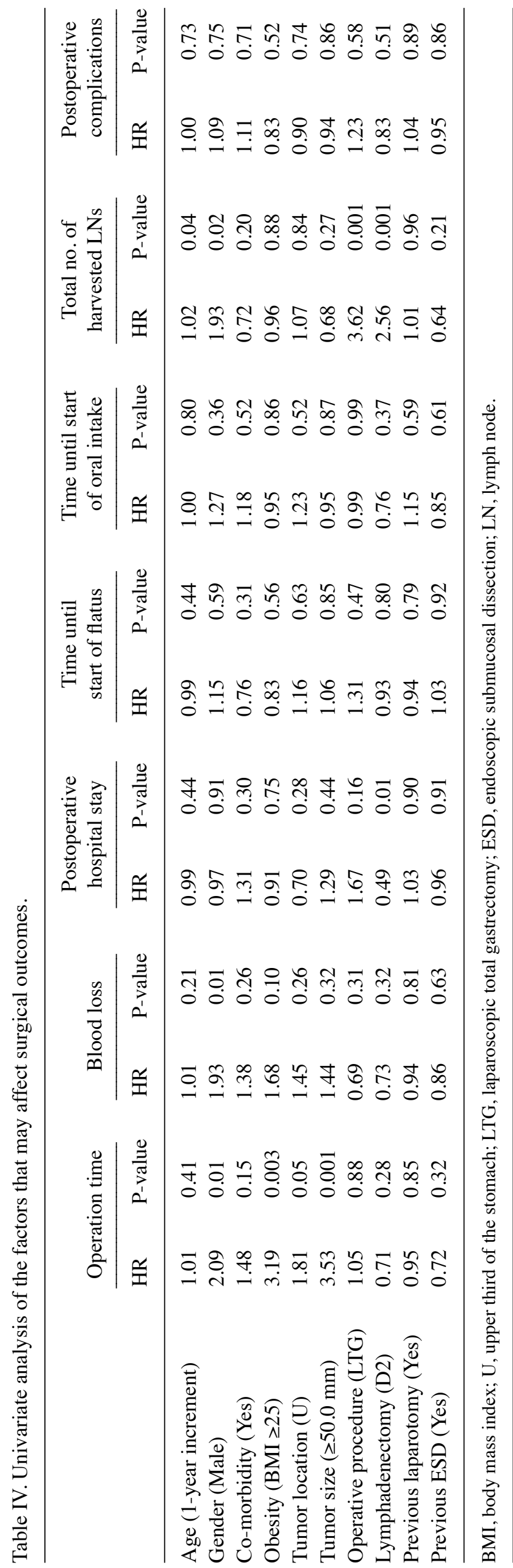

blood loss, total number of harvested lymph nodes, time until the start of flatus and the start of oral intake and postoperative hospital stay between the two groups.

We examined several factors that may affect surgical outcomes by univariate analysis (Table IV). Although several factors including gender, obesity (BMI >25), tumor size affected operation time, intraoperative blood loss, and total number of harvested lymph nodes, previous ESD did not affect any surgical outcome measure. We obtained similar results in the multivariate analysis (data not shown).

Recurrence was not observed in any patient after LAG during a mean follow-up period of 12.9 months (range, 6-25 months).

\section{Discussion}

In this study, we did not observe any surgical difficulties for LAG after previous ESD in terms of surgical outcomes such as operation time, intraoperative blood loss, total number of harvested lymph nodes, time until the start of flatus and postoperative hospital stay. In addition, these results were not influenced by tumor location and operative procedures.

It is apparent that ER causes burn, fibrosis, and adhesion of the stomach and surrounding tissue because of electrical coagulation (15). Although we experienced non-physiological adhesion around the tumor in patients with previous ESD, we did not encounter critical difficulties such as severe adhesions and anatomical misidentification during LAG. According to the univariate and multivariate analyses, previous ESD did not affect surgical outcomes such as operation time, intraoperative blood loss, postoperative hospital stay, time until the start of flatus, time until the start of oral intake, total number of harvested lymph nodes and postoperative complications. Nunobe et al (16) demonstrated that previous laparotomy, which also causes intra-abdominal adhesions, did not affect surgical outcomes and postoperative complications. This may be because of the advances in the LAG technique and the sealing devices used for LAG $(17,18)$.

Jiang et al (11) demonstrated a significantly higher rate of preservation of the celiac branch of the vagus nerve and shorter postoperative stay in patients who underwent LAG more than 2 months after ER. In this study, the average interval between LAG and ESD was 44.8 days (range, 19-80 days). We cannot conclude what interval between ER and LAG is optimal for subsequent LAG because of the small sample size, but this should be clarified in the future.

Although frequent complications caused by ER include bleeding and perforation, this study did not include any patients who experienced perforation during ER. Perforation may lead to increased adhesion and will probably increase the difficulty for LAG (11). Further studies are warranted to clarify the effect of the endoscopic procedure on subsequent LAG.

The LAG procedure is complex, but its merits for patients include less postoperative pain, early return of bowel function, short period of hospitalization and good cosmetic result (9). In this study, pathological examinations in patients without previous ESD revealed that several cases had T2 or deeper of tumor depth and/or lymph node metastases, but this was not observed for patients with previous ESD. In this regard, LAG 
may be the first-choice radical treatment after incomplete ESD for early gastric cancer $(11,19)$.

Thus, in terms of surgical outcomes, LAG is a safe and feasible procedure for treatment of early gastric cancer regardless of previous endoscopic treatment, and previous ER should not be a contraindication to LAG for early gastric cancer. As indications for ER have been extended $(20,21)$, increased numbers of patients who underwent incomplete ER should be recommended for LAG. Thus, further studies will be necessary to provide the evidence of favorable long-term feasibility and outcome.

\section{References}

1. Kim JJ, Lee JH, Jung HY, et al: EMR for early gastric cancer in Korea: a multicenter retrospective study. Gastrointest Endosc 66: 693-700, 2007.

2. Roukos DH: Current advances and changes in treatment strategy may improve survival and quality of life in patients with potentially curable gastric cancer. Ann Surg Oncol 6: 46-56, 1999.

3. Lee HJ, Yang HK and Ahn YO: Gastric cancer in Korea. Gastric Cancer 5: 177-182, 2002

4. Tsujimoto H, Sugasawa H, Ono S, Ichikura T, Yamamoto J and Hase K: Has the accuracy of preoperative diagnosis improved in cases of early-stage gastric cancer? World J Surg 34: 1840-1846, 2010.

5. Itoh H, Oohata $Y$, Nakamura $K$, Nagata T, Mibu R and Nakayama F: Complete ten-year postgastrectomy follow-up of early gastric cancer. Am J Surg 158: 14-16, 1989.

6. Lee HJ, Kim YH, Kim WH, et al: Clinicopathological analysis for recurrence of early gastric cancer. Jpn J Clin Oncol 33 : 209-214, 2003

7. Nakajima T: Gastric cancer treatment guidelines in Japan Gastric Cancer 5: 1-5, 2002.

8. Kitano S, Iso Y, Moriyama M and Sugimachi K: Laparoscopyassisted Billroth I gastrectomy. Surg Laparosc Endosc 4: 146-148, 1994.

9. Kitano S, Shiraishi N, Uyama I, Sugihara K and Tanigawa N: A multicenter study on oncologic outcome of laparoscopic gastrectomy for early cancer in Japan. Ann Surg 245: 68-72, 2007.
10. Ziqiang W, Feng Q, Zhimin C, et al: Comparison of laparoscopically assisted and open radical distal gastrectomy with extended lymphadenectomy for gastric cancer management. Surg Endosc 20: 1738-1743, 2006

11. Jiang X, Hiki N, Yoshiba H, et al: Laparoscopy-assisted gastrectomy in patients with previous endoscopic resection for early gastric cancer. Br J Surg 98: 385-390, 2011.

12. Japanese Classification of Gastric Carcinoma. 2nd English Edition. Gastric Cancer 1: 10-24, 1998.

13. Uyama I, Sugioka A, Matsui H, et al: Laparoscopic side-toside esophagogastrostomy using a linear stapler after proximal gastrectomy. Gastric Cancer 4: 98-102, 2001.

14. Inaba K, Satoh S, Ishida Y, et al: Overlap method: novel intracorporeal esophagojejunostomy after laparoscopic total gastrectomy. J Am Coll Surg 211: 25-29, 2011.

15. Tanaka M, Ono H, Hasuike N and Takizawa K: Endoscopic submucosal dissection of early gastric cancer. Digestion 77: 23-28, 2008.

16. Nunobe S, Hiki N, Fukunaga T, et al: Previous laparotomy is not a contraindication to laparoscopy-assisted gastrectomy for early gastric cancer. World J Surg 32: 1466-1472, 2008.

17. Tokunaga M, Hiki N, Fukunaga T, et al: Quality control and educational value of laparoscopy-assisted gastrectomy in a highvolume center. Surg Endosc 23: 289-295, 2009.

18. Tokunaga M, Hiki N, Fukunaga T, Nunobe S, Ohyama S and Yamaguchi T: Laparoscopy-assisted gastrectomy for patients with earlier upper abdominal open surgery. Surg Laparosc Endosc Percutan Tech 20: 16-19, 2010.

19. Tonouchi H, Mohri Y, Kobayashi M, Tanaka K, Ohi M and Kusunoki M: Laparoscopy-assisted distal gastrectomy with laparoscopic sentinel lymph node biopsy after endoscopic mucosal resection for early gastric cancer. Surg Endosc 21: 1289-1293, 2007.

20. Gotoda T, Yanagisawa A, Sasako M, et al: Incidence of lymph node metastasis from early gastric cancer: estimation with a large number of cases at two large centers. Gastric Cancer 3: 219-225, 2000.

21. Oda I, Saito D, Tada M, et al: A multicenter retrospective study of endoscopic resection for early gastric cancer. Gastric Cancer 9: 262-270, 2006. 